OPEN ACCESS

Edited by:

Xingxia Ma,

Chinese Academy of Forestry, China

Reviewed by:

Paavo Penttilä,

Aalto University, Finland

Jinzhen Cao,

Beijing Forestry University, China

Shujun Li,

Northeast Forestry University, China

*Correspondence:

Rebecca E. Ibach

rebecca.e.ibach@usda.gov

Nayomi Z. Plaza

nayomi.plazarodriguez@usda.gov

Specialty section:

This article was submitted to

Forest Management,

a section of the journal

Frontiers in Forests and Global

Change

Received: 12 November 2021

Accepted: 10 December 2021

Published: 11 January 2022

Citation:

Ibach RE, Plaza NZ and Pingali SV

(2022) Small Angle Neutron

Scattering Reveals Wood Nanostructural Features in Decay Resistant Chemically Modified Wood.

Front. For. Glob. Change 4:814086.

doi: 10.3389/ffgc.2021.814086

\section{Small Angle Neutron Scattering Reveals Wood Nanostructural Features in Decay Resistant Chemically Modified Wood}

\author{
Rebecca E. Ibach ${ }^{1 *}$, Nayomi Z. Plaza ${ }^{1 *}$ and Sai Venkatesh Pingali ${ }^{2}$ \\ ${ }^{1}$ Forest Products Laboratory, Forest Service, United States Department of Agriculture, Madison, WI, United States, \\ ${ }^{2}$ Neutron Scattering Division, Oak Ridge National Laboratory, Oak Ridge, TN, United States
}

While it is known that modifying the hydroxyls in wood can improve the decay resistance; what is often missing in the literature is whether these modifications alter wood nanostructure, and how these changes correlate to the improved decay resistance. Here, we used small angle neutron scattering (SANS) to probe the effects of alkylene oxide modifications on wood nanostructure. Southern pine wood samples were chemically modified to various weight percentage gains (WPG) using four different alkylene oxides: propylene oxide (PO), butylene oxide (BO), epichlorohydrin (EpH), and epoxybutene (EpB). After modification, the samples were water leached for 2 weeks to remove any unreacted reagents or homopolymers and then equilibrium moisture content $(\mathrm{EMC})$ was determined at $90 \%$ relative humidity $(\mathrm{RH})$ and $27^{\circ} \mathrm{C}$. Laboratory soil block decay evaluations against the brown rot fungus Gloeophyllum trabeum were performed to determine weight loss and biological efficacy of the modifications. To assist in understanding the mechanism, SANS was used to study samples that were fully immersed in deuterium oxide $\left(\mathrm{D}_{2} \mathrm{O}\right)$. These measurements revealed that the modifications altered the water distribution inside the cell wall, and the most effective modifications reduced the microfibril swelling and preserved the microfibril structure even after being subject to 12 weeks of brown rot exposure.

Keywords: SANS, solid wood modification, alkylene oxides, fungal decay, epoxybutene, propylene oxide, butylene oxide, epicholorohydrin

\section{INTRODUCTION}

Outdoor exposure of non-durable wood species often results in wood decay. Brown rot fungi, in particular, are responsible for a large part of the timber decayed in service and these affect all wood polymers but target primarily the wood carbohydrates (Goodell et al., 2020). While the mechanisms behind brown rot degradation are still being actively investigated, most evidence suggests that reducing the moisture content $(\mathrm{MC})$ of the wood is key to prevent and/or delay the onset of decay (Ringman et al., 2019; Brischke and Alfredsen, 2020). One way to reduce the $\mathrm{MC}$ of the wood is to modify it with chemical reagents that can react with the available hydroxyls to form stable, covalent bonds (Rowell, 2013). However, since most chemical modifications have 
been developed empirically over decades, the development of new chemistries is hindered by an incomplete understanding of the nanoscale interactions that lead to decay resistance.

Modifying solid wood and wood fibers with alkylene oxides such as epoxybutene (EpB), butylene oxide (BO), propylene oxide (PO), and epichlorohydrin $(\mathrm{EpH})$ can prevent weight loss caused by brown rot fungal attack with different degrees of biological efficacy (Rowell and Gutzmer, 1975; Rowell et al., 1979; Rowell and Ellis, 1984; Enoki et al., 1990; Chen, 1994; Ibach and Rowell, 2000; Ibach and Lee, 2002; Ibach and Plaza, 2019). In general, it is thought that alkylene oxides attach to the available wood hydroxyl groups and that this modification makes the modified wood less susceptible to decay (Rowell and Ellis, 1984). EpB, and $\mathrm{BO}$ have similar chemistries and have been shown to provide increased protection by lowering the moisture content of the chemically modified wood cell walls (Ibach and Plaza, 2019), but $\mathrm{EpB}$ has a $\mathrm{C}=\mathrm{C}$ that could be used for incorporating new chemistries such as UV protection. Whereas EpH attaches to the wood hydroxyls and provides adequate protection by modifying the lignocellulosic substrate without significantly lowering the sample's MC (Ibach and Lee, 2002). On the other hand, PO despite being similar to $\mathrm{EpH}$ except for the additional $\mathrm{Cl}$ molecule only slightly reduces the MC of solid wood and it does not provide significant protection from brown rot (Ibach et al., 2000). Energy-dispersive X-ray analysis (EDXA) has shown that these epoxides are able to infiltrate the cell wall, however, the extent of the infiltration and whether they can modify the nanostructure is still unknown (Ibach and Lee, 2002). The mechanisms behind decay resistance of alkylene oxide modified woods are still unclear, even though this knowledge could inform the development of new wood protection chemistries.

While probing wood nanostructure continues to be difficult, small angle neutron scattering (SANS) has become a valuable tool in lignocellulosic research due to its ability to study wood nanostructure with minimal sample preparation. Using SANS, researchers have measured changes in the average size of the microfibrils (bundles of elementary fibrils) (Penttilä et al., 2020a) and changes in the interfibrillar spacing between cellulose elementary fibrils in unmodified wood (Fernandes et al., 2011; Plaza et al., 2016; Penttilä et al., 2020b; Thomas et al., 2020) and wood modified by different chemicals including phenolformaldehyde (Plaza et al., 2019), polyethylene-glycol (Penttilä et al., 2020a) and polymethylmethacrylate (Chen et al., 2021). More recently, SANS has also been used to study how the wood nanostructure is degraded by the brown rot fungus Gloeophyllum trabeum (Goodell et al., 2017; Zhu et al., 2020) in terms of changes observed in the microfibril structure and the formation of re-polymerized lignin aggregates. Yet, despite its potential to provide new insights on how wood protection treatments alters the wood nanostructure, the technique is underutilized in this research area.

Here, we use SANS to determine how chemical modifications meant to impart decay resistance modify wood nanostructure, and how these changes affect the nanostructural degradation of the cell wall. Our findings show that those modifications effective at preventing weight loss due to brown rot fungal attack were effective at keeping the moisture outside of the cellulose microfibrils and preserved the wood nanostructural features even after 12 weeks of brown rot exposure.

\section{MATERIALS AND METHODS}

\section{Wood Modification With Alkylene Oxides}

Kiln dried southern pine (Pinus spp.) sapwood blocks (25.4 mm wide $\times 25.4 \mathrm{~mm}$ long (transverse plane) $\times 6.4 \mathrm{~mm}$ thick along the longitudinal axis) were modified with one of the following alkylene oxides: butylene oxide (BO), propylene oxide (PO), epichlorohydrin (EpH), and epoxybutene (EpB). BO and $\mathrm{EpB}$ were from Eastman Chemical Company (Kingsport, $\mathrm{TN}$, United States) and $\mathrm{PO}, \mathrm{EpH}$, and triethylamine (the catalyst) were from Aldrich Chemical Company (Milwaukee, WI, United States). All reactions were performed inside a Parr reactor using a mixture consisting of 95\% alkylene oxide, 5\% triethylamine catalyst under nitrogen. The temperature was raised gradually to 110 or $120^{\circ} \mathrm{C}$ and 150 psi (Rowell and Gutzmer, 1975; Rowell and Ellis, 1984) to control the exothermic reaction. Blocks were reacted (up to $6 \mathrm{~h}$ ) to achieve different weight percentage gains (WPG). Specimens were air-dried under a fume hood overnight prior to oven drying at $105^{\circ} \mathrm{C}$ for $24 \mathrm{~h}$. The samples were then water leached for 2 weeks to remove any unreacted reagents and homopolymers (AWPA, 1999). The average weight gain caused by the chemical modifications was determined from the oven dried weights of the individual blocks.

\section{Equilibrium Moisture Content (EMC)}

Equilibrium moisture content of unmodified and chemically modified wood samples was determined by placing weighed, oven dried samples in a constant humidity room conditioned at $90 \%$ relative humidity $(\mathrm{RH})$ and $27^{\circ} \mathrm{C}$. After $10-14$ days samples were reweighed until stable, and the EMC was determined. Six replicates of each treatment were run and averaged.

\section{Brown Rot Fungal Exposure}

The ASTM D 1413 standard soil block test was performed on oven dried solid wood southern pine specimens (ASTM, 1999). Five soil bottles each of unmodified controls and modified specimens were exposed to the brown rot fungus Gloeophyllum trabeum (MAD 617) (USDA-NRS-FMHC, Forest Products Laboratory, Madison, WI, United States). Two soil bottles each with no fungus were included to monitor leaching of any unreacted reagents or homopolymers. Specimens were removed from the test following the standard 12 weeks exposure in an environmental chamber set at $26.7^{\circ} \mathrm{C}$ and $70 \% \mathrm{RH}$. After removal, the specimens were brushed free of mycelia, air dried overnight, and then oven dried for $24 \mathrm{~h}$. The extent of decay was determined as oven dry weight loss (WL). To determine the weight loss solely caused by the brown rot exposure, the no-fungus weight loss was subtracted from all other weight loss measurements to remove any contributions caused by the leaching of any chemicals during the 12 -week test. This test was performed on unleached and water-leached samples. 


\section{Small Angle Neutron Scattering (SANS)}

For the SANS measurements, a subset of the wood blocks with different WPGs and WL were selected to determine the effects of alkylene oxide modification and brown rot fungal exposure. To prepare the samples for SANS, all these wood blocks were first soaked in water and then cut across the wood grain to expose long longitudinal surfaces from which thin longitudinal sections (less than $1 \mathrm{~mm}$ thick) were obtained. These sections were then air-dried at room temperature under a fume hood over 2 days and inspected for curling prior to being shipped to the Oak Ridge National Laboratory (ORNL) for the SANS remote experiments. A description of all samples tested using SANS is listed in Table 1, including their WPGs and/or WL from decay. Measurements were conducted at the Bio-SANS instrument in the High Flux Isotope Reactor (HFIR) facility of ORNL (Heller et al., 2014). Thin samples were immersed $\mathrm{D}_{2} \mathrm{O}$ for over $12 \mathrm{~h}$ to ensure maximizing filling of all accessible pores with $\mathrm{D}_{2} \mathrm{O}$ solvent to facilitate maximum exchange of all exchangeable hydrogens to ultimately reduce the amount of incoherent background scattering. Then, the samples were loaded into $1 \mathrm{~mm}$ path length titanium cells and filled with $\mathrm{D}_{2} \mathrm{O}$ to remove any air bubbles. The neutron incident wavelength was $6 \AA$, with a relative wavelength spread of $13.2 \%$ for the measurement. A single configuration was employed with the main detector at $15.5 \mathrm{~m}$, and the curved west wing detector fixed at $1.13 \mathrm{~m}$ from sample was rotated to $1.4^{\circ}$. This single dual detector configuration accessed a wide dynamic q-range of $0.003-0.85 \AA^{-1}$. The $2 \mathrm{D}$ SANS patterns were normalized by monitor counts and corrected for dark current, pixel sensitivity, solid angle, and background. Then, SANS data were anisotropically reduced and merged using the $d r t$-sans reduction package provided at the beamline. The reduction resulted in two azimuthally averaged sectors, one from the isotropic scattering contributions and one from the strongly aligned scattering features (anisotropic sector). The typical size for the strong anisotropic sectors was $20^{\circ}$. To track the effects of the chemical modification and/or brown rot exposure on the packing of the cellulose elementary fibrils, the datasets from the strong anisotropic sectors were fitted to a model consisting of two power laws and a Gaussian diffraction peak (Eq. 1).

$$
I(q)=B_{1} q^{-P_{1}}+B_{2} q^{-P_{2}}+A e^{-\frac{\left(q-q_{0}\right)^{2}}{\sigma^{2}}}+B_{3}
$$

For some of the more heavily modified samples (such as $\mathrm{PO} 2, \mathrm{EpB} 2$, and $\mathrm{BO} 2$ ), a scattering shoulder feature was detected within the q-range of $0.015-0.035 \AA^{-1}$, thus, a Guinier feature was added to the model to account for this contribution (Eq. 2) (Beaucage, 1995).

$$
\begin{array}{r}
I(q)=B_{1} q^{-P_{1}}+G e^{-\frac{q^{2} R_{g}^{2}}{3}}+B_{2}\left(\frac{\operatorname{erf}\left(\frac{q R_{g}}{\sqrt{6}}\right)^{3}}{q}\right)^{P_{2}} \\
+A e^{-\frac{\left(q-q_{0}\right)^{2}}{\sigma^{2}}}+B_{3}
\end{array}
$$

Scattering from the cellulose microfibrils might become more

\begin{tabular}{|c|c|c|}
\hline Sample description & $\begin{array}{l}\text { Modification level } \\
\text { WPG (\%) }\end{array}$ & $\begin{array}{c}\text { Decay level WL } \\
(\%)\end{array}$ \\
\hline Unmodified wood (UW) & 0 & - \\
\hline $\begin{array}{l}\text { UW exposed to brown rot fungi } \\
\text { (UW-BRE) }\end{array}$ & 0 & 66.5 \\
\hline $\begin{array}{l}\text { Wood lightly modified with butylene oxide } \\
\text { (BO1) }\end{array}$ & -0.2 & - \\
\hline Wood modified with butylene oxide (BO2) & 14.8 & - \\
\hline $\begin{array}{l}\text { BO1 wood exposed to brown rot fungi } \\
\text { (BO1-BRE) }\end{array}$ & -0.1 & 50.8 \\
\hline $\begin{array}{l}\text { BO2 wood exposed to brown rot fungi } \\
\text { (BO1-BRE) }\end{array}$ & 15.5 & 22.5 \\
\hline $\begin{array}{l}\text { Wood lightly modified with propylene } \\
\text { oxide (PO1) }\end{array}$ & 1.3 & - \\
\hline $\begin{array}{l}\text { Wood modified with propylene oxide } \\
\text { (PO2) }\end{array}$ & 21.0 & - \\
\hline $\begin{array}{l}\text { P01 wood exposed to brown rot fungi } \\
\text { (P01-BRE) }\end{array}$ & 1.4 & 46.9 \\
\hline $\begin{array}{l}\text { PO2 wood exposed to brown rot fungi } \\
\text { (PO2-BRE) }\end{array}$ & 23.6 & 30.9 \\
\hline $\begin{array}{l}\text { Wood lightly modified with epoxybutene } \\
\text { (EpB1) }\end{array}$ & $10.1^{*}$ & - \\
\hline Wood modified with epoxybutene (EpB2) & $36.2^{*}$ & - \\
\hline $\begin{array}{l}\text { EpB1 wood exposed to brown rot fungi } \\
\text { (EpB1-BRE) }\end{array}$ & 5.8 & 25.0 \\
\hline $\begin{array}{l}\text { EpB2 wood exposed to brown rot fungi } \\
\text { (EpB2-BRE) }\end{array}$ & 31.6 & -0.4 \\
\hline $\begin{array}{l}\text { Wood lightly modified with } \\
\text { epichlorohydrin (EpH1) }\end{array}$ & 2.8 & - \\
\hline $\begin{array}{l}\text { Wood modified with epichlorohydrin } \\
(\mathrm{EpH} 2)\end{array}$ & 13.0 & - \\
\hline $\begin{array}{l}\text { EpH1 wood exposed to brown rot fungi } \\
\text { (EpH1-BRE) }\end{array}$ & 6.7 & 23.2 \\
\hline $\begin{array}{l}\text { EpH2 wood exposed to brown rot fungi } \\
\text { (EpH2-BRE) }\end{array}$ & 12.2 & 5.6 \\
\hline
\end{tabular}
prominent in this range. This model was also used to fit the
TABLE 1 | Description of samples measured using SANS.

*Water-leached samples were unavailable, so unleached samples were tested instead.

scattering from samples that exhibited some weight loss due to the brown rot fungal exposure. For these decayed samples the Guinier feature was necessary to account for the contribution of a mid-q shoulder feature that has been previously attributed to the formation of repolymerized lignin (Goodell et al., 2017; Zhu et al., 2020). All data analyses were performed in IgorPro8 using the Modeling II tools included in the Irena package (Ilavsky and Jemian, 2009). Data beyond $0.5 \AA^{-1}$ was not included in the fitting range due to an instrument artifact observed in all measurements around $q=0.5 \AA^{-1}$. Representative datasets fitted with Eqs. 1, 2 are plotted in Supplementary Figures 1, 2, respectively, to highlight the individual contributions of the low$\mathrm{q}$, mid- $\mathrm{q}$ and high- $\mathrm{q}$ regions to the overall fit.

\section{RESULTS}

For a chemical modification of solid wood to be considered biologically effective the weight loss caused by the 12 -week fungal exposure should be less than $5 \%$. Weight losses between 5 to 
$30 \%$ correspond to partially effective modifications and larger than $30 \%$ are considered ineffective. Modifying solid wood with the alkylene oxides $\mathrm{EpB}, \mathrm{EpH}$, and $\mathrm{BO}$ protected the wood from brown rot degradation to different degrees but were all effective at the highest WPGs. PO, on the other hand, did not sufficiently reduce the weight loss caused by brown rot exposure (BRE) to be considered effective. In general, the weight loss (WL) due to the fungal attack was reduced with increasing WPG (Figure 1). $\mathrm{EpB}$ and $\mathrm{EpH}$ were the most effective at reducing the weight loss, and even at low modification levels (WPG $<14 \%$ ) the weight loss was reduced to less than $5 \%$ compared to the $65 \%$ WL observed for the unmodified wood samples. Water leaching the samples removed any water-soluble extractives or unreacted epoxides left from the modification, and as a result both the weight loss and the WPG were lower compared to the unleached samples. This was most evident for the EpH water leached samples, which had much lower weight loss after the 12-week fungal exposure compared to the unleached samples. The EMC of the modified wood decreased with increasing WPG compared to the unmodified wood for BO and $\mathrm{EpB}$ modified samples, whereas for $\mathrm{PO}$ and $\mathrm{EpH}$ the EMC did not change significantly with increasing WPG (Figure 1).

Using SANS, we studied a subset of samples with different degrees of modification and decay to further understand the nanostructural origins of the differences observed. Figure $\mathbf{1}$ shows the average values and standard deviations of all the samples, whereas Table 1 shows the WPG and WL from decay values of the individual samples analyzed with SANS.

\section{Nanostructure of Alkylene Oxide Modified Wood}

For unmodified wood (UW) immersed in $\mathrm{D}_{2} \mathrm{O}$, the contrast between water-accessible regions and non-water accessible regions inside the wood cell walls gives rise to distinct anisotropic scattering profiles that include power law scattering from the bundles of the cellulose microfibrils as well as other wood polymers, and a characteristic high-q diffraction peak ( $q \sim 0.14 \AA^{-1}$ ) that is caused by the regular packing of the cellulose elementary fibrils. Modifying the wood with alkylene oxides is expected to reduce the hydrophilic nature of the wood polymers inside the cell wall by grafting an epoxide onto the available hydroxyl groups in the wood polymers (Rowell and Ellis, 1984) without forming any byproducts that could not be water-leached. Thus, there is very little difference between the scattering length density (SLD), which is a measure of neutron sensitivity of the molecule, of the unmodified wood polymers
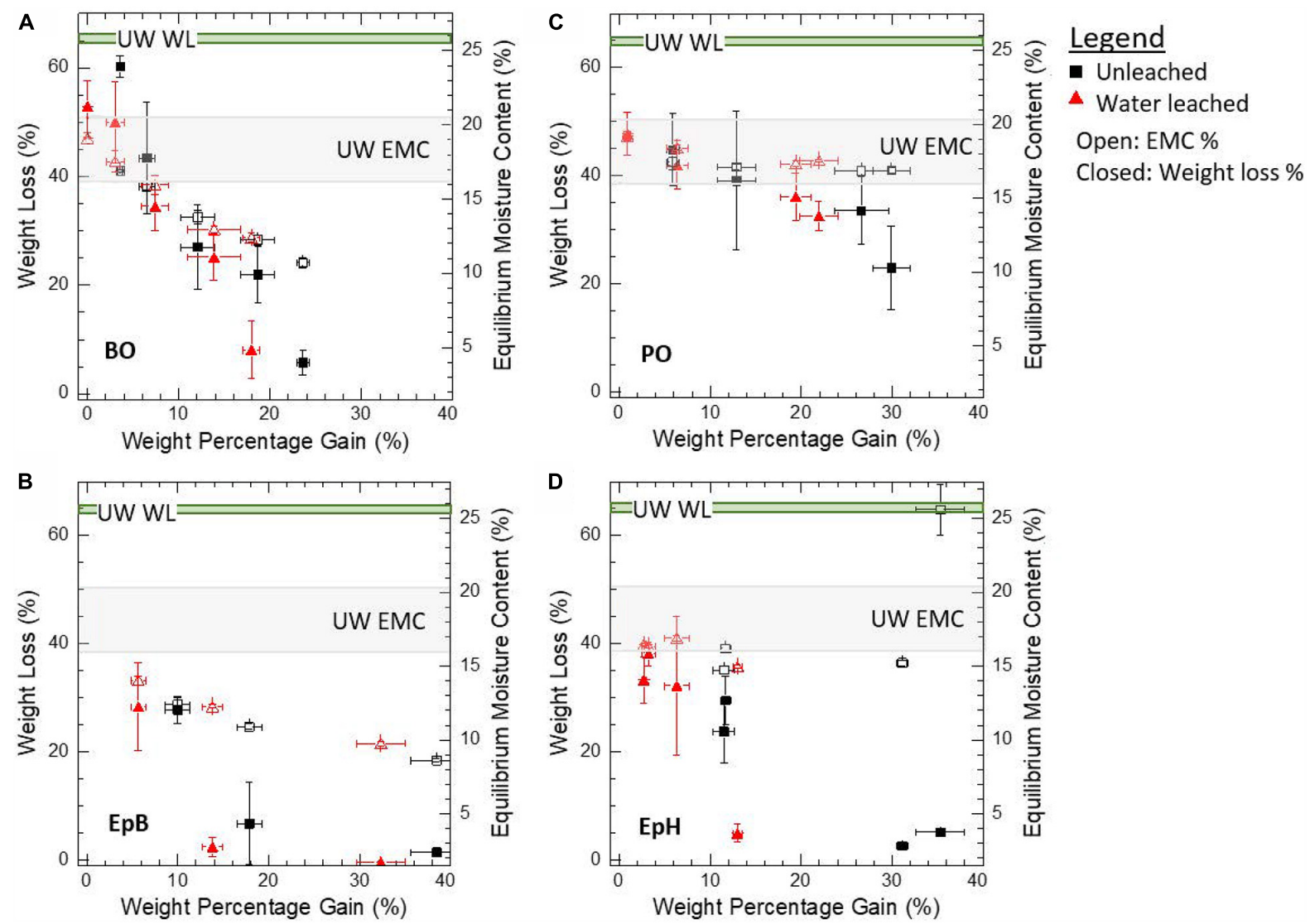

FIGURE 1 | Effect of modification level, in terms of weight percentage gain, on the weight loss caused by brown rot fungal exposure after 12 weeks (left $y$-axis) and the average $\mathrm{EMC}$ at $90 \% \mathrm{RH}$ and $27^{\circ} \mathrm{C}$ after modification (right $y$-axis). Samples were modified with (A) butylene oxide (BO), (B) epoxybutene (EpB), (C) propylene oxide (PO), and (D) epichlorohydrin (EpH). Weight loss was corrected for non-fungal weight loss contributions. Unmodified wood (UW) values are also shown. 
with respect to those modified by the alkylene oxides (SLD of $1.87 \times 10^{-6} \AA^{-2}$ for cellulose vs. $1.1-1.6 \times 10^{-6} \AA^{-2}$ for a modified wood polymer). Therefore, when modified or unmodified wood samples are immersed in $\mathrm{D}_{2} \mathrm{O}$ (SLD of $6.3 \times 10^{-6} \AA^{-2}$ ) the source of contrast giving rise to the scattering is the same (water-accessible regions vs. non-water accessible). Despite this similarity, we observe some distinct differences in the scattering profiles of the alkylene oxide modified woods with respect to the unmodified wood (Figure 2). For instance, when the sample is modified with $\mathrm{EpB}, \mathrm{EpH}$ or $\mathrm{BO}$ we observe that the diffraction peak $\left(q \sim 0.14 \AA^{-1}\right.$ for UW) shifts to higher $\mathrm{q}\left(\mathrm{q} \sim 0.18 \AA^{-1}\right)$, meaning that the cellulose microfibril is swelling less in the modified wood compared to the unmodified one. Whereas for PO the position of the diffraction peak is unaffected by the modification implying wood modified with PO is unable to resist the swelling observed for unmodified wood samples. Moreover, modifying the wood with high levels of $\mathrm{EpB}, \mathrm{BO}$ or $\mathrm{PO}$ gives rise to a weak mid$\mathrm{q}$ shoulder feature $\left(\mathrm{q} \sim 0.03 \AA^{-1}\right)$ that is not observed in the scattering profile from unmodified wood. Interestingly, the inflection where the shoulder is observed is comparable in size to the diameter of cellulose microfibrils (bundle of elementary fibrils) observed in secondary cell walls from softwoods using scanning electron microscopy (Donaldson, 2007; Lyczakowski et al., 2019), atomic force microscopy (Adobes-Vidal et al., 2020) and SANS (Penttilä et al., 2020b; Zhu et al., 2020). This observation implies $\mathrm{D}_{2} \mathrm{O}$ solvent is unable to penetrate between some of the elementary fibrils and therefore produce features of the cellulose microfibrils. Conversely, we do not observe this new mid-q shoulder feature in the EpH modified wood, which indicates that this modification is uniformly modifying the wood structure without changing the nanoscale water distribution inside the cell wall even at the highest level of modification studied.

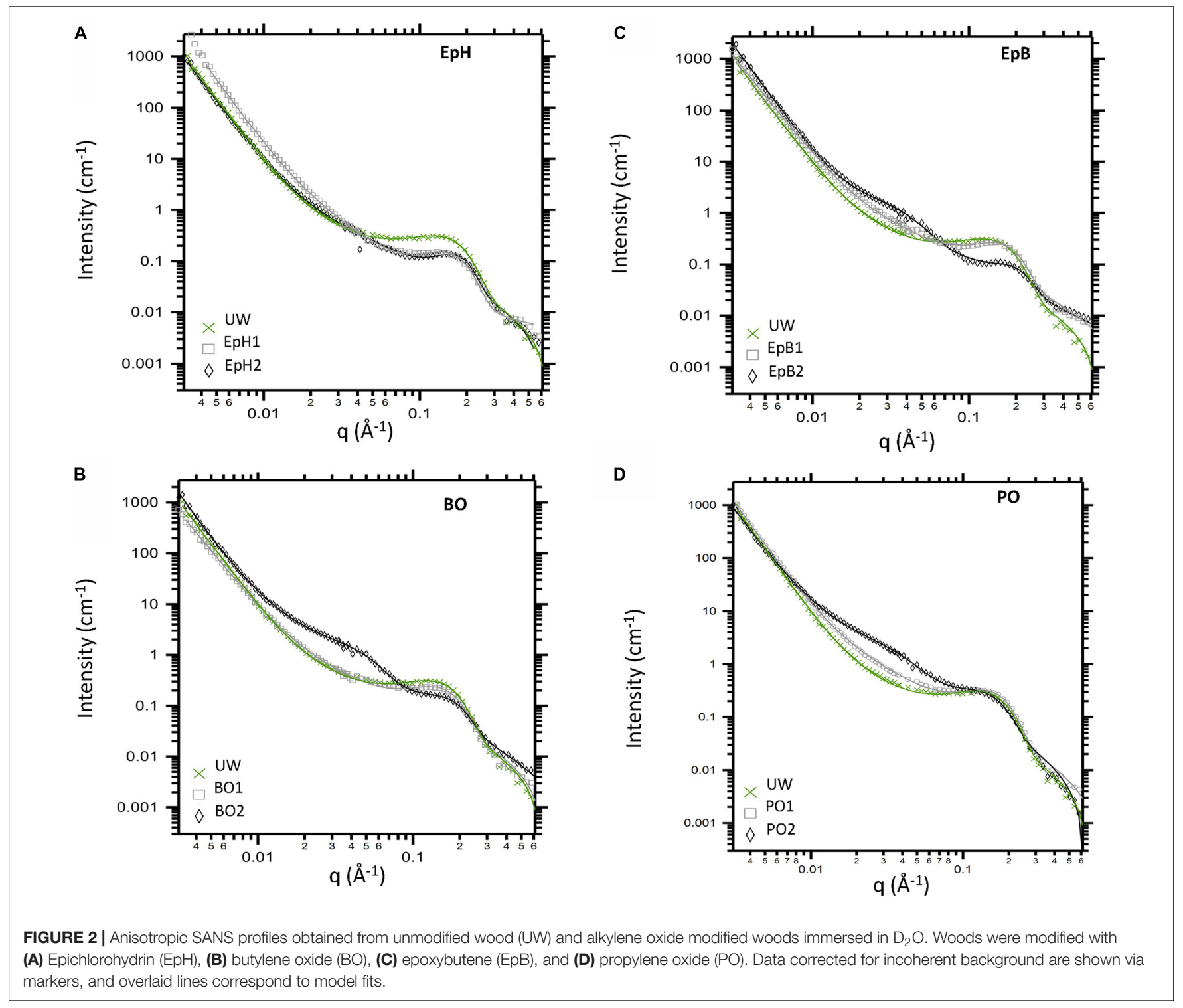


By fitting each anisotropic profile, we tracked the effects of the chemical modifications on the wood nanostructure. The parameters describing the low-q and mid-q scattering regions are listed in Table 2. Chemically modifying the wood did not considerably change the low-q power law scattering. For most samples, we observed a power law exponent close to $\sim 4$, which indicates that the low-q scattering $\left(q<0.015 \AA^{-1}\right)$ is dominated by scattering from relatively smooth surfaces of large-sized structures. The mid-q region is typically the most complex to interpret due to the overlapping contributions of scattering from the wood polymer matrix as well as scattering from the crosssection of the bundles of the cellulose elementary fibrils, which are quite polydisperse in size. However, it is possible that as the relative amount of water outside the microfibrils increases due to the modification, the scattering contribution of the larger sized microfibrils dominates and their scattering contribution becomes more prominent in our scattering data. For most samples, the mid-q power law exponents ranged from 1.4 to 2.3 , with the higher exponents being observed for the chemically modified samples. Power law exponents close to 1 correspond to scattering from rod-like particles such as the elementary fibrils in the unmodified wood, and values closer to 2 typically correspond to scattering from flexible polymer conformations. Hence, the increase observed in the mid-q power law exponent (from 1.4 to $\sim 2$ ) could be attributed to the initial swelling of the wood polymer network caused by the chemical modification since all these chemicals swell wood to different extents (Rowell and Ellis, 1984). Larger power law exponents between 2 and 3 correspond to mass fractal scattering, thus, for the highly modified PO and EpB, the mid-q power law exponent observed could be attributed to scattering from less-water accessible regions inside the cell wall that are formed by the modification. For highly modified PO, BO, and EpB wood, we also observed a broad Guinier region, whose radius of gyration $\left(\mathrm{R}_{\mathrm{g}}\right)$ ranges between 5 to $10 \mathrm{~nm}$, with the largest $\mathrm{R}_{\mathrm{g}}$ being observed for PO (Table 2). The similarities between $\mathrm{EpB}$ and $\mathrm{BO}$ would suggest that these modifications are interacting similarly with the wood polymers, whereas PO interacts differently. Considering that we observed this contribution in both the anisotropic scattering and the

TABLE 2 | Chemical modifications effects on the low and mid-q range scattering parameters.

\begin{tabular}{lcccc}
\hline Sample & $\begin{array}{c}\text { Low } \mathbf{q} \\
\mathbf{q}<\mathbf{0 . 0 1 5} \AA^{-1}\end{array}$ & & Mid-q $\mathbf{0 . 0 1 5} \AA^{-\mathbf{1}}<\mathbf{q}<\mathbf{0 . 0 8 5} \AA^{-\mathbf{1}}$ \\
\cline { 2 - 3 } & $\mathbf{P}_{\mathbf{1}}$ & & $\mathbf{P}_{\mathbf{2}}$ & $\mathbf{R}_{\mathbf{g}}$ (nm) \\
\cline { 2 - 3 } & $4.3+/-0.09$ & & $1.4+/-0.1$ & - \\
UW & $3.9+/-0.07$ & & $1.4+/-0.1$ & - \\
BO1 & $4.01+/-0.07$ & & $1.8+/-0.25$ & $6+/-0.97$ \\
BO2 & $4.01+/-0.05$ & & $1.5+/-0.1$ & - \\
PO1 & $3.8+/-0.11$ & & $2.2+/-0.03$ & $10+/-0.6$ \\
PO2 & $4.04+/-0.08$ & & $1.6+/-0.1$ & - \\
EpB1 & $4.11+/-0.05$ & & $2.3+/-0.04$ & $5+/-0.5$ \\
EpB2 & $4.33+/-0.08$ & & $1.7+/-0.1$ & - \\
EpH1 & $4.24+/-0.08$ & & $1.6+/-0.1$ & - \\
EpH2 & & &
\end{tabular}

isotropic scattering profiles, it is also possible that the reaction between the wood polymers and these alkylene oxides leads to the formation of some hydrophobic wood polymer aggregates, and thus, their size starts to contribute to the mid-q scattering. However, if this was the sole contributor of the mid-q shoulder feature, one would expect larger differences between EpB2 and $\mathrm{BO} 2$ based on their different WPGs (36WPG for EpB2 vs. $\sim 15 \mathrm{WPG}$ for $\mathrm{BO} 2$ ). In the future, contrast variation studies will be pursued to determine the source of the mid-q shoulder feature observed.

The effects of the chemical modification on the packing of the cellulose elementary fibrils are shown in Figure 3. In general, we observe that higher WPGs led to smaller elementary fibril spacings except for PO. At low levels of modification (WPGs < 10\%) the spacing between the elementary fibrils was comparable to the unmodified samples, but at higher WPGs less water was able to infiltrate the cellulose microfibrils, and the spacing between the elementary fibrils decreased and became comparable to the packing expected in dry cellulose microfibrils even though the samples were fully immersed in $\mathrm{D}_{2} \mathrm{O}$. The relative decrease in deuterated water content inside the cellulose microfibrils led to a decrease in the contrast between the elementary fibril and its surrounding, which consequently, increased the uncertainty in the evaluation of the peak position as evidenced by the larger error bars. Interestingly, if we compare the behavior of the elementary fibril spacing with the EMC at $90 \% \mathrm{RH}$ as a function of the WPG, we observe that these trends have similar shape for all except PO, where the EMC was relatively constant as a function of WPG but the elementary fibril spacing $\left(\mathrm{EF}_{\mathrm{s}}\right)$ still increased. It is conceivable that some of these alkylene oxides can penetrate the cellulose microfibrils and occupy some of the spaces between the cellulose elementary fibrils and thus, reduce their ability to uptake water and swell

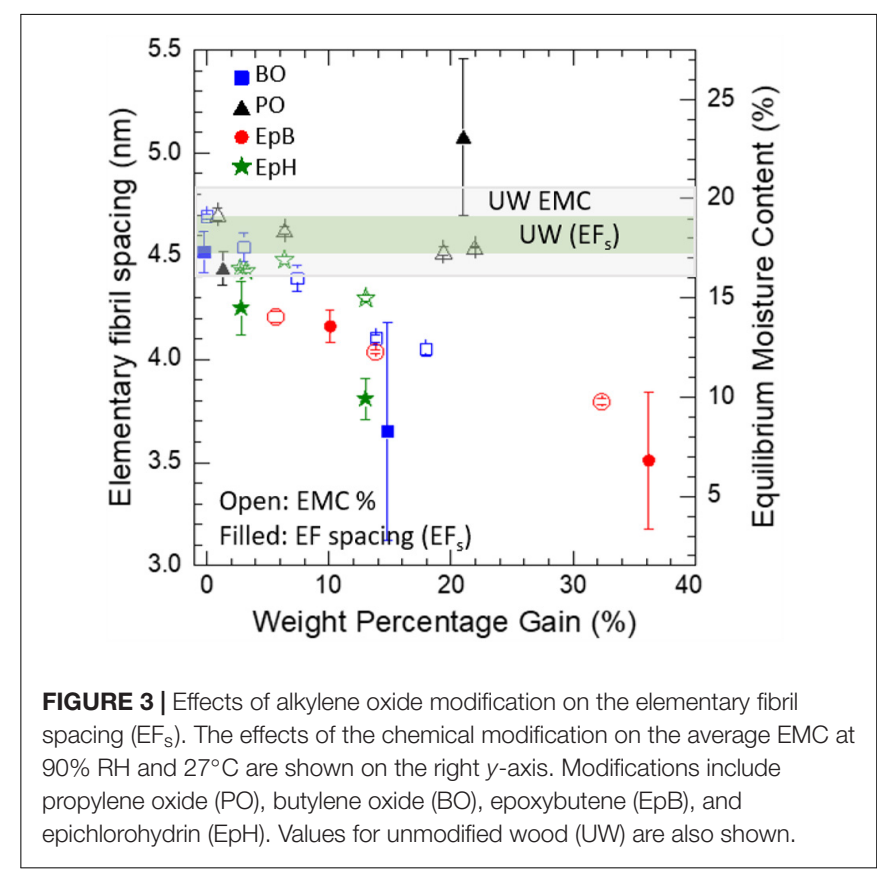


even when immersed in $\mathrm{D}_{2} \mathrm{O}$. However, in these experiments we do not have enough contrast between the modified wood polymers and the unmodified wood polymers to determine if all alkylene oxides tested can infiltrate the microfibril. The larger more irregular spacing between elementary fibrils observed for PO would suggest that PO had a pre-swelling effect on some of the microfibrils and increased the microfibril initial size but did not prevent sub-sequent swelling and thus the elementary fibril spacing observed for PO samples was larger than the one observed for unmodified wood. However, it is also possible that the increase in elementary fibril spacing occurred due to the coalescence of some elementary fibrils as the PO modified the matrix polymers where the microfibrils are embedded. If the $\mathrm{PO}$ is interacting more strongly with the matrix polymers it is conceivable that some aggregate is being formed due to the modification and its size is contributing strongly to the increased mid-q scattering observed.

\section{Nanostructure of Alkylene Oxide Modified Wood Exposed to Brown Rot Fungus}

Once the unmodified wood had been exposed to the brown rot fungus, the structural integrity of the sample was compromised due to degradation, (Figures $\mathbf{4 A}$ vs. $\mathbf{B}$ ); and as a result, the scattering profiles show loss of some of its characteristic diamond-like shape at low $\mathrm{q}$ and becomes much more isotropic across all length scales (Figures 4E,I vs. F,J). For alkylene oxide modified samples like PO which became brittle and suffered weight loss after fungal exposure (Figures 4C vs. D), it was difficult to obtain intact sections from brown rot exposed samples, and thus these samples also exhibited less anisotropic patterns (Figures $\mathbf{4 G}, \mathbf{K}$ vs. $\mathbf{H}, \mathbf{L}$ ). Non-uniform alignment between individual wood sections inside the titanium cell would have also contributed to reduced anisotropic scattering.

By comparing the SANS profiles from the samples before and after exposure to brown rot, we observe that decay resistant modified woods like $\mathrm{EpH}$ and $\mathrm{EpB}$ and $\mathrm{BO}$, all preserved the high-q diffraction peak (Figure 5) while for PO modified wood this feature was nearly indistinguishable. Also, wood lightly modified with BO was not as effective at preserving the wood nanostructure. The brown-rot exposure led to a mid-q shoulder feature observed in all samples that exhibited some weight loss, making it difficult to determine whether the mid-q shoulder feature observed in the $\mathrm{BO} 2$ and $\mathrm{PO} 2$ samples was preserved from the fungal attack. For the heavily modified EpB, however, a mid$\mathrm{q}$ shoulder feature is present in the brown-rot exposed sample even though it did not suffer any weight loss indicating that this modification preserved both mid-q and high-q nanostructural features. No mid-q shoulder feature was observed for decay resistant $\mathrm{EpH}$ modified wood even after the 12-week exposure. Thus, treatments that were able to considerably reduce the weight loss due to brown rot exposure were also able to protect the wood nanostructural features.

The effects of the brown rot exposure on the scattering parameters describing the low-q and mid-q regions of the SANS profiles are shown in Table 3. At the low q, we mostly observed

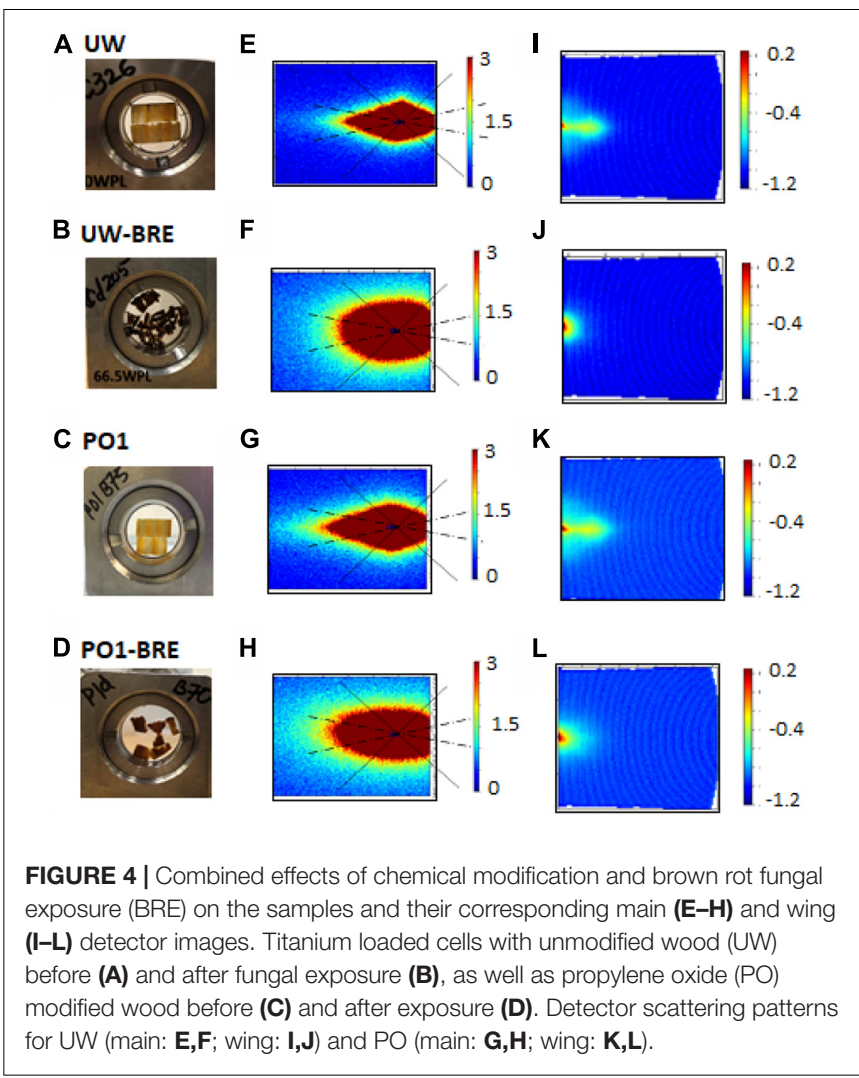

scattering from relatively smooth surfaces that led to a power law exponent of $\sim 4$. The brown rot exposure increased the roughness of these surfaces, and as a result the low-q power law exponent decreased, as observed previously by others (Goodell et al., 2017; Zhu et al., 2020). For samples that were only chemically modified, the scattering contribution from the swollen wood polymer network is the major contributor to the mid-q scattering region. However, once these samples had been exposed to the brown rot fungus, we observed the following changes in the midq scattering region: an increase in the power law exponent, and a broad shoulder feature that is not observed in non-BRE samples. These changes indicate that there is a new feature contributing to the mid-q scattering. The mid-q power law exponent ranged between $\sim 2.2$ and $\sim 2.9$, which corresponds to scattering from mass fractals, and the radius of gyration $\left(R_{\mathrm{g}}\right)$ ranged between 8 and $10 \mathrm{~nm}$. These mass fractals have been previously attributed to the formation of lignin agglomerates that are caused due to the repolymerization of lignin by the brown rot fungi. Interestingly, the $\mathrm{R}_{\mathrm{g}}$ of these agglomerates resulting from the brown-rot exposure did not change significantly between modified samples that suffered weight loss even for the $\mathrm{BO} 2$ and $\mathrm{PO} 2$ samples, which exhibited a mid-q shoulder feature due to the modification. While it is possible that the mid-q shoulder feature observed in $\mathrm{PO} 2$ and $\mathrm{BO} 2$ became less pronounced due to the brown rot fungal attack, it is difficult to determine whether this feature was still present in the BRE samples. For the heavily modified $\mathrm{EpB}$, which did not exhibit any weight loss due to the brown rot exposure, the mid-q shoulder feature caused by the modification 

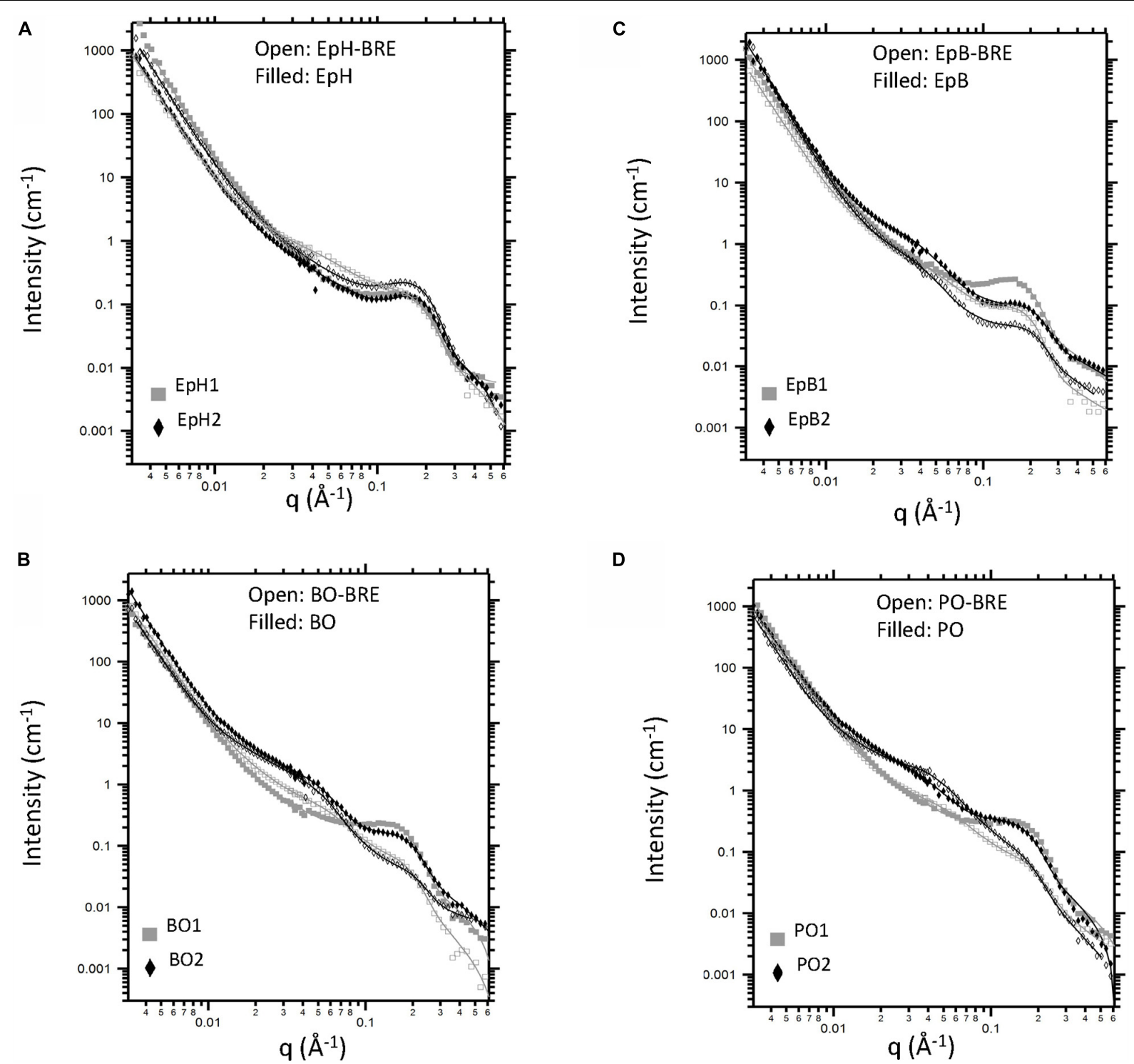

FIGURE 5 | Effects of alkylene oxide modification and brown rot fungal exposure (BRE) on wood nanostructure. Anisotropic scattering profiles (corrected for incoherent background) from unmodified wood (UW) and wood modified with (A) epichlorohydrin (EpH), (B) epoxybutene (EpB), (C) butylene oxide (BO), and (D) propylene oxide (PO). Filled symbols correspond to data from samples that were only chemically modified. Open symbols correspond to samples that were both chemically modified and exposed to brown rot fungi. Model fits are shown as overlaid lines.

was still present and the associated $\mathrm{R}_{\mathrm{g}}$ was comparable to the one obtained from the unexposed sample, meaning that this feature was preserved from the fungal attack implying EpB was hindering brown rot access to the cellulose microfibril.

The combined effects of chemical modification and brown rot fungal exposure on the elementary fibril spacing $\left(\mathrm{EF}_{\mathrm{s}}\right)$ are shown in Figure 6. All modifications reduced the microfibril swelling with increasing WPG, except for PO, which was also the only ineffective modification. After brown rot fungal exposure, for most samples the intensity of the diffraction peak decreased, which increased the uncertainty of the associated peak position in the decayed samples compared to the non-decayed ones. Nevertheless, the $\mathrm{EF}_{\mathrm{s}}$ were unaffected by brown rot exposure, except for EpH. For heavily modified $\mathrm{EpH}$, the $\mathrm{EF}_{\mathrm{s}}$ increased after brown rot exposure and became comparable to those measured for the unmodified sample. At lower WPGs $(<10 \%)$, the differences in the WPG of the individual EpH samples are likely responsible for the differences observed in the $\mathrm{EF}_{\mathrm{S}}$.

\section{DISCUSSION}

Treatments with alkylene oxide altered the moisture-induced swelling of the cellulose microfibrils before and after brown rot exposure differently, suggesting that these chemicals can target different regions in the wood cell walls and thus, there 
TABLE 3 | Effects of brown rot fungi exposure on the low and mid-q scattering parameters.

\begin{tabular}{|c|c|c|c|}
\hline \multirow[t]{2}{*}{ Sample } & \multirow{2}{*}{$\frac{\text { Low q }}{q<0.015 \AA^{-1}}$} & \multicolumn{2}{|c|}{ Mid-q $0.015 \AA^{-1}<q<0.085 \AA^{-1}$} \\
\hline & & $\mathbf{P}_{2}$ & $\mathbf{R}_{\mathrm{g}}(\mathrm{nm})$ \\
\hline UW-BRE & $4.0+/-0.1$ & $2.3+/-0.3$ & $8+/-1.2$ \\
\hline BO1-BRE & $3.7+/-0.0$ & $2.5+/-0.5$ & $7+/-1.5$ \\
\hline BO2-BRE & $3.9+/-0.1$ & $2.3+/-0.1$ & $7.7+/-0.02$ \\
\hline PO1-BRE & $3.71+/-0.03$ & $2.3+/-0.3$ & $7+/-1.4$ \\
\hline PO2-BRE & $3.9+/-0.09$ & $2.9+/-0.1$ & $7.7+/-0.02$ \\
\hline EpB1-BRE & $3.89+/-0.05$ & $2.4+/-0.5$ & $8+/-1.8$ \\
\hline EpB2-BRE & $4.4+/-0.08$ & $2.2+/-0.02$ & $6+/-0.42$ \\
\hline EpH1-BRE & $3.87+/-0.06$ & $2.2+/-0.3$ & $8+/-1.5$ \\
\hline EpH2-BRE & $4.0+/-0.2$ & $1.8+/-0.1$ & $10+/-0.4$ \\
\hline
\end{tabular}

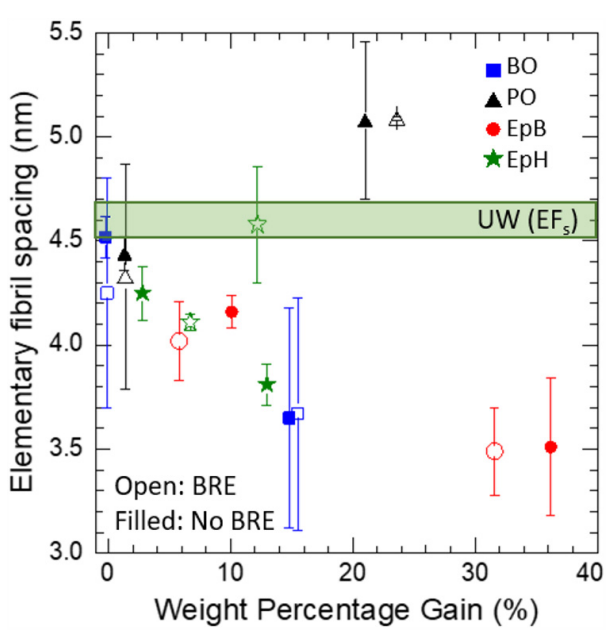

FIGURE 6 | Combined effects of alkylene oxide modification and brown rot exposure $(\mathrm{BRE})$ on the elementary fibril spacing $\left(\mathrm{EF}_{\mathrm{S}}\right)$. Error bars corresponds to the uncertainty in the diffraction peak position with respect to variations in the fitting parameters, so the peak position of weaker diffraction peaks will have larger error bars. Modifications included: epichlorohydrin (EpH), epoxybutene (EpB), butylene oxide (BO) and propylene oxide (PO). Unmodified wood (UW) values are also shown.

are different nanoscale pathways to achieving biological efficacy. The observed nanostructural changes caused by alkylene oxide modification can be classified as (1) templating of the wood nanostructure combined with a reduction in the microfibril swelling or (2) a change in the nanoscale water distribution inside the wood cell wall that gave rise to a new mid-q shoulder feature that is not typically observed in scattering from unmodified wood.

The first type of nanostructural change was only observed in wood modified with EpH, which templated the wood nanostructure and reduced the microfibril swelling even at low levels of WPG. While all other modifications led to nanostructural changes of the second type. All biologically effective modifications, namely $\mathrm{EpB}, \mathrm{BO}$ and $\mathrm{EpH}$, reduced the amount of water entering the microfibrils, and hence reduced microfibril swelling. For $\mathrm{EpB}$ and $\mathrm{BO}$, which are chemically similar and had nearly identical scattering features, there was also an increase of water outside the microfibrils, as evidenced by the stronger mid-q scattering contributions, which suggests that these two modifications were able to change the hydrophilic nature of the wood polymers where the microfibrils are embedded in a similar way. On the other hand, PO, the least effective treatment has been shown to be more reactive in ligninrich earlywood compared to latewood (Rowell and Ellis, 1984), so it is possible that PO interacted more with the lignin in the matrix where the cellulose microfibrils are embedded. This interaction could have led to the coalescence of some elementary fibrils and increased their effective interfibrillar spacing. Alternatively, PO could have also infiltrated the microfibrils as well and caused a pre-swelling of the microfibrils that did not protect the microfibrils from brown rot fungal attack or prevented further moisture-induced swelling. Interestingly, the $\mathrm{EF}_{\mathrm{S}}$ observed in the PO modified wood is comparable to those observed in delignified wood immersed in $\mathrm{D}_{2} \mathrm{O}$ (Chen et al., 2021). Contrast variation studies will further enable elucidation of the effects of these chemical modifications on the wood polymers. Nonetheless, the differences observed in the EFs strongly suggest that these alkylene oxides are targeting different wood polymers. PO is likely interacting more strongly with the lignin-rich matrix outside the cellulose microfibrils, while $\mathrm{BO}$ and $\mathrm{EpB}$ are interacting more strongly with the polymers inside the cellulose microfibrils. $\mathrm{EpH}$, on the other hand, is uniformly modifying the wood polymers.

Interestingly, the most biologically effective treatments, namely, $\mathrm{EpH}$ and $\mathrm{EpB}$ reduced the EMC to below $16 \%$ and minimized the spacing between elementary fibrils to values comparable to those expected for a relatively dry microfibril. Previously, it has been proposed that keeping the EMC of wood below $16 \%$ is key to impede the softening of the hemicelluloses, which in turn, inhibits cell wall diffusion and decreases the susceptibility to fungal decay (Jakes et al., 2013; Jakes, 2019). Our SANS data shows for the first time that biologically effective modifications exclude moisture from the cellulose microfibrils and that the swelling of the microfibrils is correlated to the decay resistance of the samples. Moreover, our data also suggests that effective modifications can hinder and/or prevent the formation of diffusion channels inside the cellulose microfibrils.

\section{CONCLUSION}

By combining SANS with EMC analysis and fungal exposures tests, we discovered that alkylene oxide modifications that impart brown rot decay resistance also minimize the swelling of the cellulose microfibrils and preserve the overall cellulose microfibril nanostructure. These findings show for the first time that the nanoscale moisture distribution inside the cell wall plays a key role in fungal degradation by G. trabeum, and that keeping water from entering the cellulose microfibrils can prevent and/or delay fungal decay by G. trabeum. SANS can provide valuable insights on the nanostructural mechanisms behind the effectiveness of different wood chemical modifications. 


\section{DATA AVAILABILITY STATEMENT}

The Small Angle Neutron Scattering (SANS) data can be accessed at: http://doi.org/10.5281/zenodo.5589817.

\section{AUTHOR CONTRIBUTIONS}

NP developed and performed the experimental design for the nanostructural analysis. RI developed and performed the chemical modifications, as well as the fungal exposure assays and EMC analysis. SP performed the SANS experiments on the thin sections prepared by NP. NP conducted the bulk of the SANS data analysis with support from SP. All authors contributed to the writing of the manuscript.

\section{FUNDING}

This research was supported by the U.S. Department of Agriculture Forest Service.

\section{ACKNOWLEDGMENTS}

A portion of this work performed acknowledges the support of the Genomic Science Program, Office of Biological and Environmental Research (OBER), U.S. Department of Energy (DOE), under Contract FWP ERKP752. The SANS studies on Bio-SANS (IPTS-21873.1) were supported by the OBER funded

\section{REFERENCES}

Adobes-Vidal, M., Frey, M., and Keplinger, T. (2020). Atomic force microscopy imaging of delignified secondary cell walls in liquid conditions facilitates interpretation of wood ultrastructure. J. Struct. Biol. 211:107532. doi: 10.1016/j. jsb.2020.107532

ASTM (1999). ASTM standard method of testing wood preservatives by laboratory soil-block cultures. Am. Soc. Test. Mater. Des. 9:1413.

AWPA (1999). E11-97 Standard Method of Determining the Leachability of Wood Preservatives. Alabama, AL: American Wood Preservers Association, 386-388.

Beaucage, G. (1995). Approximations leading to a unified exponential/power-law approach to small-angle scattering. J. Appl. Crystallogr. 28, 717-728. doi: 10. 1107/S0021889895005292

Brischke, C., and Alfredsen, G. (2020). Wood-water relationships and their role for wood susceptibility to fungal decay. Appl. Microbiol. Biotechnol. 104, 37813795. doi: 10.1007/s00253-020-10479-1

Chen, G. C. (1994). Fungal decay resistance of wood reacted with chlorosulfonyl isocyanate or epichlorohydrin. Holzforschung 48, 181-185.

Chen, P., Li, Y., Nishiyama, Y., Pingali, S. V., O’Neill, H. M., Zhang, Q., et al. (2021). Small angle neutron scattering shows nanoscale PMMA distribution in transparent wood biocomposites. Nano Lett. 21, 2883-2890. doi: 10.1021/acs. nanolett.0c05038

Donaldson, L. (2007). Cellulose microfibril aggregates and their size variation with cell wall type. Wood Sci. Technol. 41, 443-460. doi: 10.1007/s00226-006-0121-6

Enoki, A., Yoshioka, S., Fuse, G., and Tanaka, H. (1990). Fungal and termite resistance and dimensional stability of etherified woods prepared with epichlorohydrin. J. Antibacter. Antifung. Agents 18, 431-439.

Fernandes, A. N., Thomas, L. H., Altaner, C. M., Callow, P., Forsyth, V. T., Apperley, D. C., et al. (2011). Nanostructure of cellulose microfibrils in spruce wood. Proc. Natl. Acad. Sci. U.S.A. 108, E1195-E1203. doi: 10.1073/pnas. 1108942108
Center for Structural Molecular Biology (CSMB) under Contract FWP ERKP291, using the High Flux Isotope Reactor supported by the Basic Energy Sciences, Department of Energy. The findings and conclusions in this publication are those of the authors and should not be construed to represent any official USDA or U.S. Government determination or policy. This manuscript has been coauthored by UT-Battelle, LLC, under Contract No. DEAC05- 00OR22725 with the U.S. Department of Energy. The United States Government retains and the publisher, by accepting the article for publication, acknowledges that the United States Government retains a non-exclusive, paid-up, irrevocable, worldwide license to publish or reproduce the published form of this manuscript, or allow others to do so, for United States Government purposes. The authors would like to acknowledge assistance from Beom-Goo Lee and Phillip Smith.

\section{SUPPLEMENTARY MATERIAL}

The Supplementary Material for this article can be found online at: https://www.frontiersin.org/articles/10.3389/ffgc.2021. 814086/full\#supplementary-material

Supplementary Figure 1 | Anisotropic scattering profile from wood lightly modified with epoxybutene (EpB1) showing the overall fit (overlaid black line), as well as the individual contributions of the low-q, mid-q, and high-q terms in Eq. 1.

Supplementary Figure 2 | Anisotropic scattering profile from wood heavily modified with epoxybutene (EpB2) showing the overall fit (overlaid black line), as well as the individual contributions of the low-q, mid-q, and high-q terms in Eq. 2.

Goodell, B., Winandy, J. E., and Morrell, J. J. (2020). Fungal degradation of wood: emerging data, new insights and changing perceptions. Coatings 10:1210. doi: 10.3390/coatings 10121210

Goodell, B., Zhu, Y., Kim, S., Kafle, K., Eastwood, D., Daniel, G., et al. (2017). Modification of the nanostructure of lignocellulose cell walls via a non-enzymatic lignocellulose deconstruction system in brown rot wood-decay fungi. Biotechnol. Biofuels 10:179. doi: 10.1186/s13068-0170865-2

Heller, W. W. T., Urban, V. S. V., Lynn, G. G. W., Weiss, K. L., O’Neill, H. M., Pingali, S. V., et al. (2014). The bio-Sans instrument at the high flux isotope reactor of oak ridge national laboratory. J. Appl. Crystallogr. 47, 1238-1246. doi: $10.1107 /$ S1600576714011285

Ibach, R. E., and Lee, B.-G. (2002). “The effect on biological and moisture resistance of epichlorohydrin chemically modified wood," in Proceedings of the 33rd Annual Meeting of the International Research Group on Wood Preservation IRG Document No: IRG/WP/02-40224, Cardiff.

Ibach, R. E., and Plaza, N. (2019). "Using X-ray scattering to elucidate the mechanisms behind the moisture and fungal decay resistance of epoxybutene modified wood," in Proceedings of the 15th Annual Meeting of the International Research Group on Wood Protection IRG/WP 19-40854, Quebec City, QC, $1-17$.

Ibach, R. E., and Rowell, R. M. (2000). Improvements in decay resistance based on moisture exclusion. Mol. Cryst. Liq. Cryst. 353, 23-33. doi: 10.1080/ 10587250008025645

Ibach, R. E., Rowell, R. M., and Lee, B.-G. (2000). "Decay protection based on moisture exclusion resulting from chemical modification of wood," in Proceedings of the 5th Pacific Rim Bio-Based Composites, Canberra, ACT, 197204.

Ilavsky, J., and Jemian, P. R. (2009). Irena: tool suite for modeling and analysis of small-angle scattering. J. Appl. Crystallogr. 42, 347-353. doi: 10.1107/ S0021889809002222 
Jakes, J. E. (2019). Mechanism for diffusion through secondary cell walls in lignocellulosic biomass. J. Phys. Chem. B 123, 4333-4339. doi: 10.1021/acs.jpcb. $9 \mathrm{~b} 01430$

Jakes, J. E., Plaza, N., Stone, D. S., Hunt, C. G., Glass, S. V., and Zelinka, S. L. (2013). Mechanism of transport through wood cell wall polymers. J. For. Prod. Ind. 2, 10-13. doi: 10.1021/bk-2004-0864.ch013

Lyczakowski, J. J., Bourdon, M., Terrett, O. M., Helariutta, Y., Wightman, R., and Dupree, P. (2019). Structural imaging of native cryo-preserved secondary cell walls reveals the presence of macrofibrils and their formation requires normal cellulose, lignin and xylan biosynthesis. Front. Plant Sci. 10:1398. doi: 10.3389/ fpls. 2019.01398

Penttilä, P. A., Altgen, M., Awais, M., Österberg, M., Rautkari, L., and Schweins, R. (2020a). Bundling of cellulose microfibrils in native and polyethylene glycolcontaining wood cell walls revealed by small-angle neutron scattering. Sci. Rep. 10:20844. doi: 10.1038/s41598-020-77755-y

Penttilä, P. A., Altgen, M., Carl, N., van der Linden, P., Morfin, I., Österberg, M., et al. (2020b). Moisture-related changes in the nanostructure of woods studied with X-ray and neutron scattering. Cellulose 27, 71-87. doi: 10.1007/s10570019-02781-7

Plaza, N. Z., Jakes, J. E., Frihart, C. R., Hunt, C. G., Yelle, D. J., Lorenz, L. F., et al. (2019). Small-angle neutron scattering as a new tool to evaluate moistureinduced swelling in the nanostructure of chemically modified wood cell walls. For. Prod. J. 68, 349-352. doi: 10.13073/FPJ-D-17-00065

Plaza, N. Z., Pingali, S. V., Qian, S., Heller, W. T., and Jakes, J. E. (2016). Informing the improvement of forest products durability using small angle neutron scattering. Cellulose 23, 1593-1607. doi: 10.1007/s10570-016-0933-y

Ringman, R., Beck, G., and Pilgård, A. (2019). The importance of moisture for brown rot degradation of modified wood: a critical discussion. Forests 10:522. doi: 10.3390/f10060522

Rowell, R. M. (2013). Handbook of Wood Chemistry and Wood Composites, 2nd Edn. Milton Park: Routledge.
Rowell, R. M., and Ellis, W. D. (1984). Reaction of Epoxides with Wood. Madison, WI: Forest Products Laboratory Report, 451.

Rowell, R. M., and Gutzmer, D. I. (1975). Chemical modification of wood: reactions of alkylene oxides with southern yellow pine. Wood Sci. 7, 240-246.

Rowell, R. M., Hart, S. V., and Esenther, G. R. (1979). Resistance of alkylene-oxide treatments on dimensional stability of wood. Wood Sci. 11:271.

Thomas, L. H., Martel, A., Grillo, I., and Jarvis, M. C. (2020). Hemicellulose binding and the spacing of cellulose microfibrils in spruce wood. Cellulose 27, 4249-4254. doi: 10.1007/s10570-020-03091-Z

Zhu, Y., Plaza, N., Kojima, Y., Yoshida, M., Zhang, J., Jellison, J., et al. (2020). Nanostructural analysis of enzymatic and non-enzymatic brown rot fungal deconstruction of the lignocellulose cell wall ${ }^{\dagger}$. Front. Microbiol. 11:1389. doi: $10.3389 /$ fmicb. 2020.01389

Conflict of Interest: The authors declare that the research was conducted in the absence of any commercial or financial relationships that could be construed as a potential conflict of interest.

Publisher's Note: All claims expressed in this article are solely those of the authors and do not necessarily represent those of their affiliated organizations, or those of the publisher, the editors and the reviewers. Any product that may be evaluated in this article, or claim that may be made by its manufacturer, is not guaranteed or endorsed by the publisher.

Copyright (C) 2022 Ibach, Plaza and Pingali. This is an open-access article distributed under the terms of the Creative Commons Attribution License (CC BY). The use, distribution or reproduction in other forums is permitted, provided the original author(s) and the copyright owner(s) are credited and that the original publication in this journal is cited, in accordance with accepted academic practice. No use, distribution or reproduction is permitted which does not comply with these terms. 